\title{
Influence of the Types of Stress on Hydrogen Induced Damage By Simulating Hydrogen Diffusion
} \author{
Shaojie Zhang ${ }^{1, a}$, Hehui Wang ${ }^{1, b}$, Feng Hou ${ }^{1, c}$, Hu Chen ${ }^{2, d}$, Pingqing Tan ${ }^{3, \mathrm{e}}$ \\ ${ }^{1}$ School of Mechanical and Power Engineering, East China University of Science and Technology, \\ Shanghai, 200237, China; \\ ${ }^{2}$ Ningbo Special Equipment Inspection Center,315048,China; \\ ${ }^{3}$ Sinopec Zhenhai Refining \& Chemical Company,315221, China.

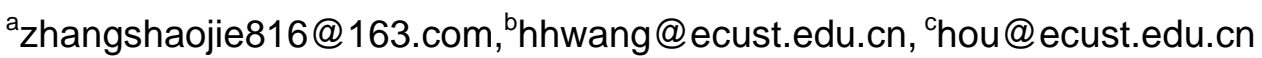 \\ ${ }^{\mathrm{d}} \mathrm{ch} \_$nbtj@163.com, ${ }^{\mathrm{e}}$ tanpq.zhlh@sinopec.com
}

Keywords: stress type, hydrogen damage, stress-assisted diffusion, finite element analysis.

\begin{abstract}
This paper describes an approach to identify the influence of different stress type on hydrogen diffusion of the specimen which contains both tensile stress and pressure stress. The numerical method of simulating hydrogen diffusion by using software Abaqus is used to determine the influence. The simulated hydrogen diffusion is based on Fick's second law. By comparing the concentration of different nodes which locate in different stress type area, it can be concluded that the tensile stress will drive the hydrogen diffusion but the pressure stress will hinder the hydrogen diffusion.
\end{abstract}

\section{Introduction}

Hydrogen uptake in steel pipes can initiate or speed up various forms of degradation, such as external internal cracks, which will develop to be a blister [1]. Atomic hydrogen can be dissolved in the steel as a consequence of manufacturing process or environment. If there are corrosion phenomena on the steel surface, it will produce hydrogen atoms which can diffusion into the steel lattice [2]. There are two different diffusion mechanisms, first is vacancy diffusion, another is interstitial diffusion. The vacancy diffusion mechanism is atoms exchange with vacancies, it is applied to substitutional impurity atoms, its rate depends on the number of vacancies and activation energy to exchange. The interstitial diffusion is that the smaller atoms $(\mathrm{H}, \mathrm{C}, \mathrm{O}, \mathrm{N})$ can diffuse between atoms which is bigger than hydrogen atom and so on. It is more rapid than vacancy diffusion duo to more mobile atoms and more empty interstitial sites. Compared with the metallic atoms, the hydrogen atom is very small so that they can diffuse through the interstitial sites. Diffused hydrogen will be trapped at different micro structural features [3]. The diffused hydrogen atoms collected in trapping sites will recombine to form hydrogen gas which will generate high pressure [4]. With time and corrosion occurrence, the hydrogen pressure will become higher and can easily lead to the nucleation of microcracks, which finally coalesce leading to catastrophic failures, even at stresses which below the yield stress [5]. There is three different hydrogen induced damage: (i) Hydrogen Induced Cracking (HIC), (ii) Stress Oriented HIC (SOHIC) and (iii) hydrogen blistering. HIC is characterized by cracking which can be accompanied by through-thickness cracks. SOHIC is usually the results of the coalescence of HIC cracks. The blister is caused by hydrogen accumulation at inclusions. The recombination of atomic hydrogen to hydrogen gas generates high local stresses in the crack cavity, which can eventually exceed the yield stress of the material thereby leading to plastic defor1mation and surface blister [6].

When the crack cavity is full of local hydrogen gas pressure, stress gradient will be generated between the base metal and inclusions. The stress gradient may make the hydrogen atoms diffuse toward to the region which is full of high stress. The collected hydrogen atoms will increase over time, which will lead to the increase of the hydrogen pressure according to Sievert s law. The stress gradient also may make the hydrogen atoms diffuse away from the region which is full of high 
stress. Diffusion of hydrogen will be simulated by using software Abaqus/Standard so that we can study which type of stress can promote the diffusion or impede the diffusion of hydrogen atom.

\section{Numerical simulations}

The specimen material was 20R. The finite element model is shown in Fig. 1. The elements of region of the crack tip are refinement. Before the analysis of diffusion hydrogen, the stress analysis should be conducted in order to obtain the stress filed distribution. The stress analysis model is shown in Fig. 2, a normal pressure is applied along the crack interface and the constraints are applied along the lateral side. The diffusion analysis model is the same as the stress analysis model except the boundary conditions. The inner wall of the diffusion model is charged at a constant hydrogen concentration (100ppm) which depends on the chemical composition of the sour environment. The concentration of the outer surface is assumed to be zero. The diffusion simulation adopt DC2D4 element.

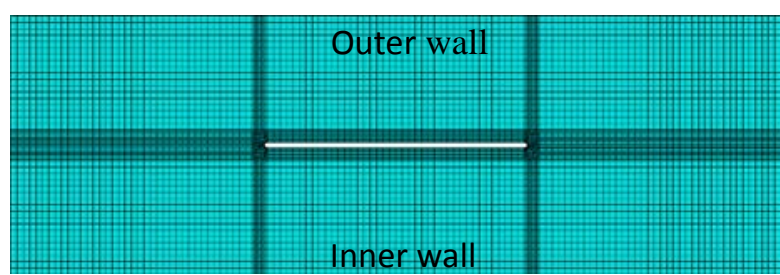

Fig. 1 Finite element model.

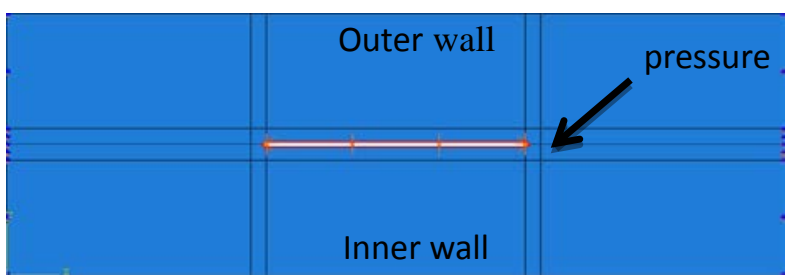

Fig. 2 The loading model of stress analysis

During the stress analysis, different groups of pressure which is $10 \mathrm{MPa}, 20 \mathrm{Mpa}, 30 \mathrm{MPa}$, $40 \mathrm{MPa}$, respectively, is applied. The pressure applied on the crack inner surface was used to simulate hydrogen pressure which produced by collected hydrogen gas inside the crack. The influence of different stress magnitude on hydrogen diffusion can be studied.

Material model. The material model is based on the Fick's second law [7] as is shown in equation (1). During the process of hydrogen diffusion, the hydrogen atom diffusion can be affected by various factors, such as the stress, temperature and some other material self-factor like solubility and diffusivity. In a short, the hydrogen diffusion constitutive equation as is shown in equation (2) is adopted.

$$
\begin{aligned}
& \frac{\partial C}{\partial t}=D \frac{\partial^{2} C}{\partial x^{2}} \\
& J=-s D \cdot\left[\frac{\partial \phi}{\partial x}+k_{s} \frac{\partial}{\partial x}\left(\ln \left(\theta-\theta^{z}\right)\right)+k_{\sigma} \frac{\partial \sigma}{\partial x}\right]
\end{aligned}
$$

Where D is diffusivity; $s$ is the solubility; $k_{\mathrm{s}}$ is the "Soret effect" factor, providing diffusion because of temperature gradient; $\theta$ is the temperature; $\theta^{\mathrm{Z}}$ is the value of absolute zero temperature; $\mathrm{k}_{\mathrm{p}}$ is the pressure stress factor. The parameters of the finite element model are shown in table 1.

Table 1. Parameters of the finite element model.

\begin{tabular}{cccc}
\hline Parameter & Id & Description & Value \\
\hline Geometric & $\mathrm{L}$ & length & $30.0 \mathrm{~mm}$ \\
Specimen & $\mathrm{h}$ & Thickness & $10.0 \mathrm{~mm}$ \\
Material & $\mathrm{E}$ & Yong's modulus & $200[\mathrm{GPa}]$ \\
Elastic & $\mathrm{u}$ & Poisson's Ratio & 0.3 \\
Diffusion & $\mathrm{D}$ & Hydrogen Diffusivity & $2.32 \times 10^{-3} \mathrm{~mm}^{2} / \mathrm{s}$ \\
& $\mathrm{s}$ & Hydrogen Solubility & $2.48 \times 10^{-4} \mathrm{ppm}$ \\
& $\mathrm{T}$ & Temperature & $323 \mathrm{~K}$ \\
\hline
\end{tabular}




\section{Results and discussion}

The result of the stress analysis is shown in Fig. 3. The local stress of the crack tip is very large, much larger than stress of surrounding crack area. The stress of gray area exceeds 100MPa. With applied pressure increase, the stresses also increase.

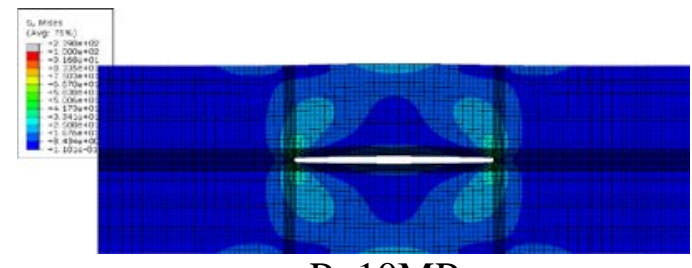

$\mathrm{P}=10 \mathrm{MPa}$

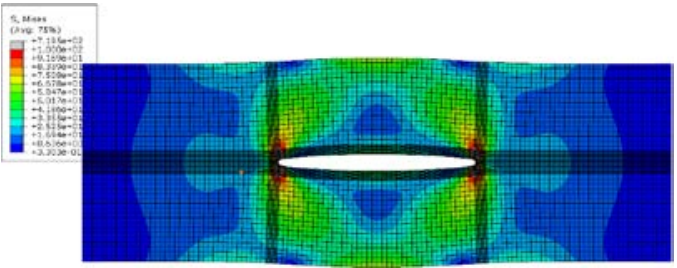

$\mathrm{P}=30 \mathrm{MPa}$

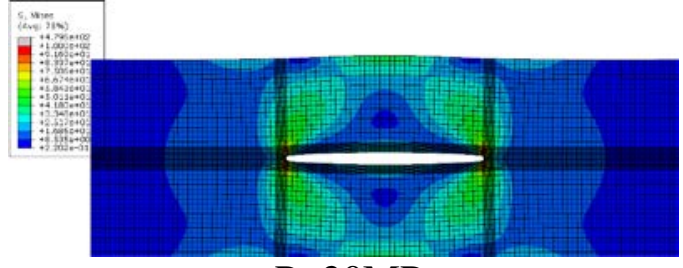

$\mathrm{P}=20 \mathrm{MPa}$

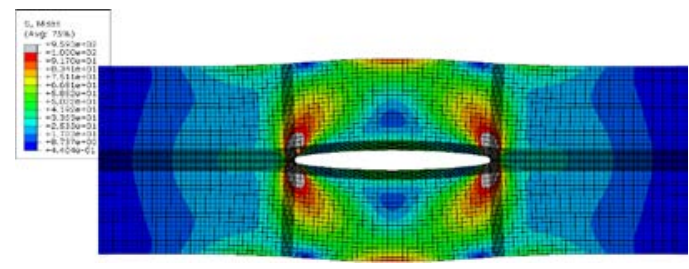

$\mathrm{P}=40 \mathrm{MPa}$

Fig. 3 The Mises stress of specimen under different pressure

After stress analysis, stress field will be used as initial condition coupled with hydrogen diffusion. The coupled stress-diffusion result is shown in Fig. 4. The concentration of gray area exceeds 1000ppm. The concentration curve of crack tip front node is shown in Fig. 5. It can see from Fig. 5 with increase of stress of crack tip, the concentration of crack tip front also increase. The stress type of crack tip front is tensile stress. It can be concluded that the tensile stress contribute to the hydrogen diffusion.

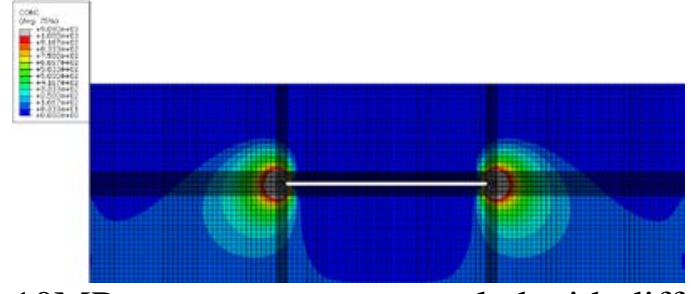

10MPa pressure stress coupled with diffusion

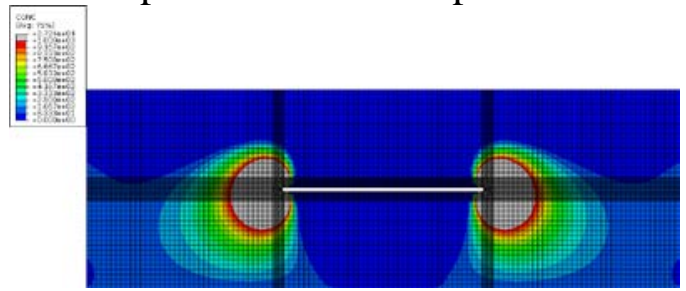

30MPa pressure stress coupled with diffusion

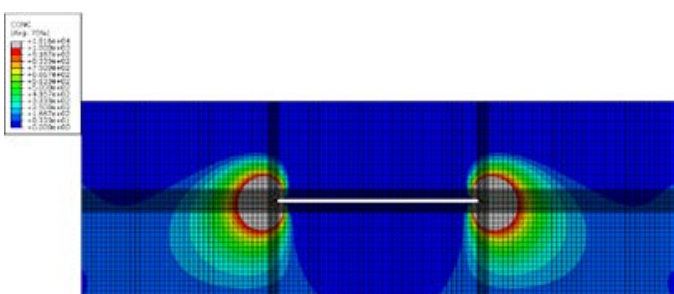

20MPa pressure stress coupled with diffusion

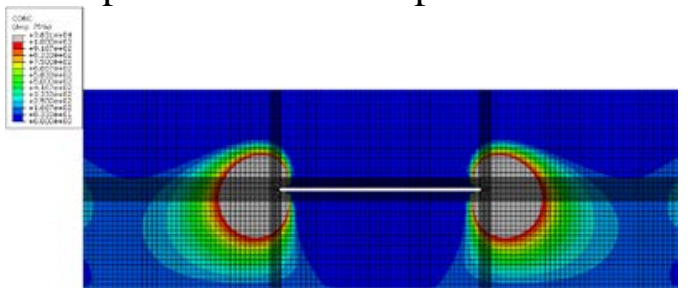

40MPa pressure stress coupled with diffusion nder condition of stress coupled with diffusion

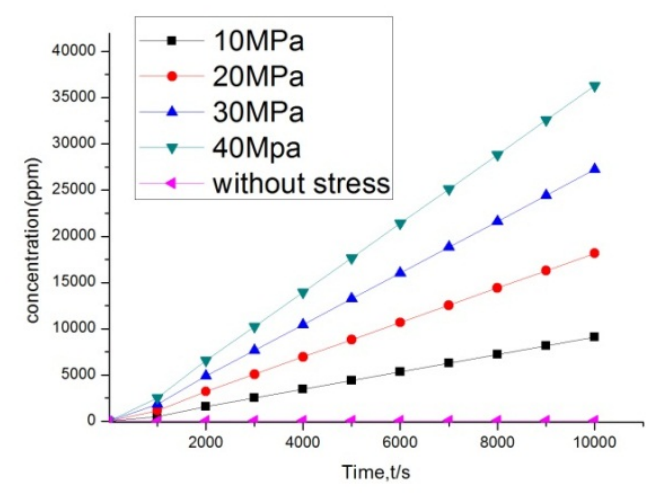

Fig. 5 Concentration curve of crack tip node

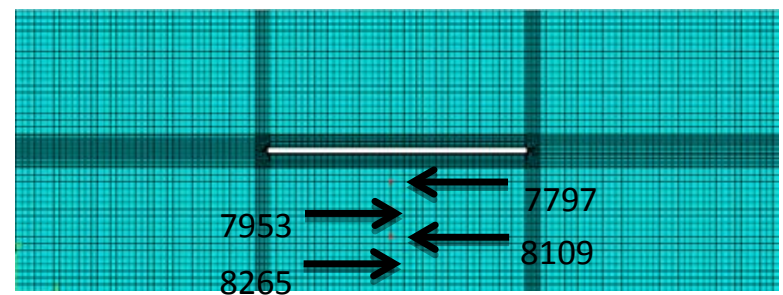

Fig. 6 The location of four nodes in specimen 
Four nodes are selected in the specimen, which is 8265, 8109, 7953 and 7797, respectively, as is shown in Fig. 6. Extract the stress of four nodes and make a chart, as is shown in Fig. 7. From the Fig. 7 we can conclude that the four nodes are all in pressure stress state. The pressure stress of the node which is close to crack boundary is bigger than that of the node which away from crack boundary.

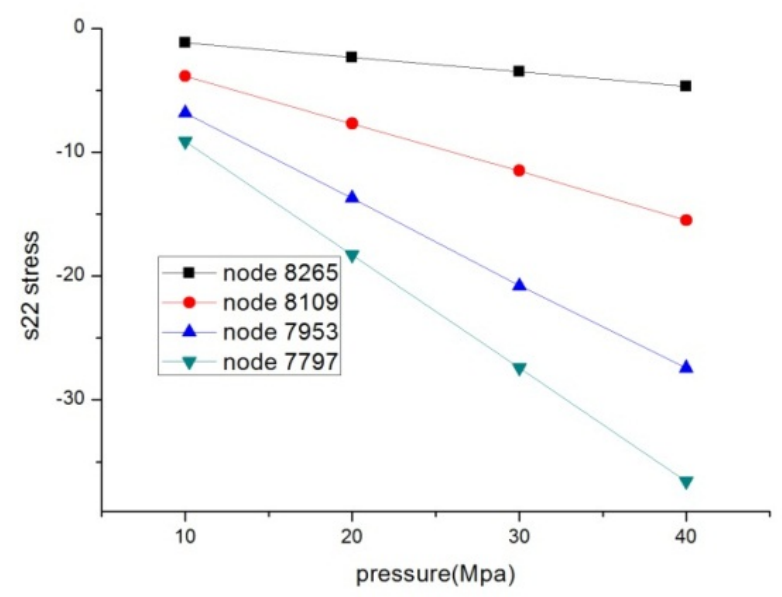

Fig. 7 Pressure stress of four nodes under different pressure

Fig. 8 shows that the variation trend of curve about node concentration presents parabolic type. Hydrogen concentration increases over time during the early stages of the hydrogen diffusion. After the concentration reaches a maximum, it will decrease over time. Make a contrast of different node we can know that the maximum concentration of the node which under higher pressure stress is smaller than that of the node which under lower pressure. It can be concluded that the pressure stress hinder the diffusion of hydrogen atoms in the specimen.

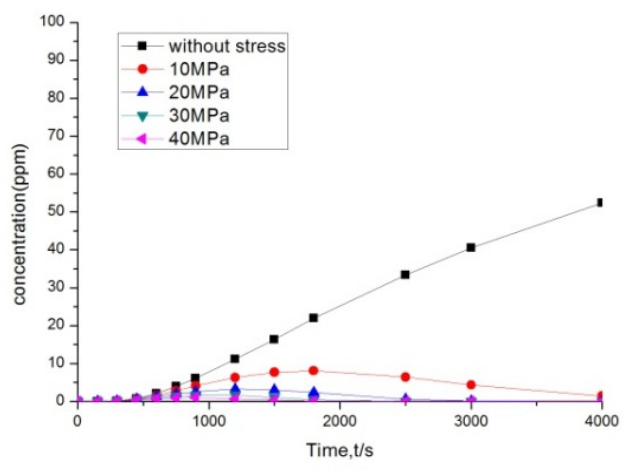

Node 7797

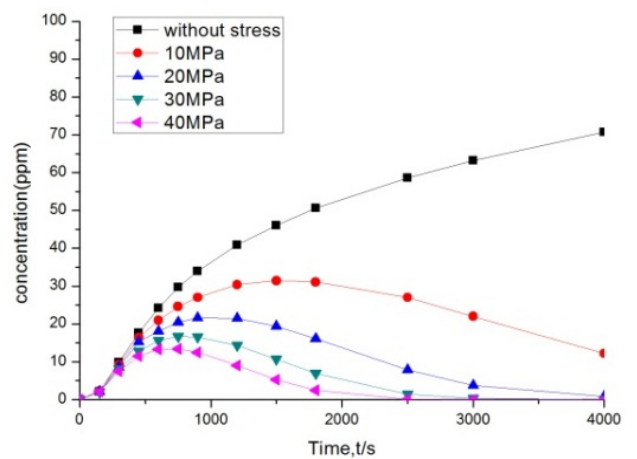

Node 8109

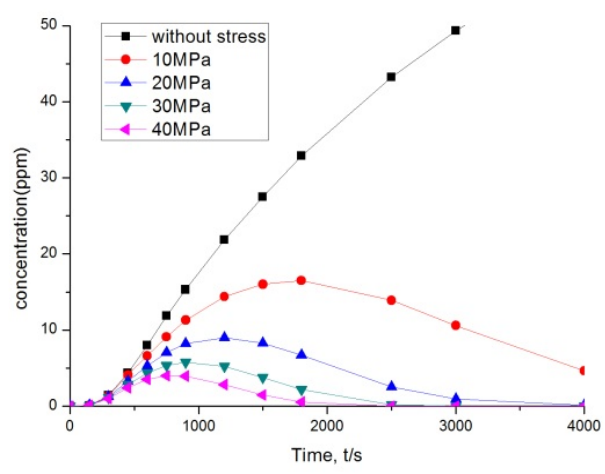

Node 7953

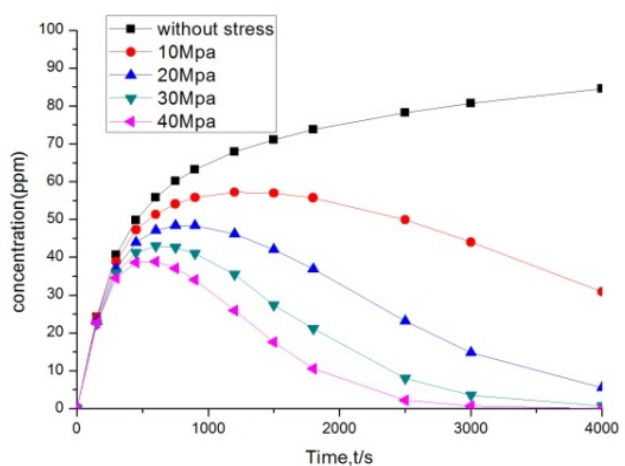

Node 8265

Fig. 8 The concentration curves of nodes under different pressure 


\section{Conclusion}

There are many factors influencing the hydrogen diffusion, stress is also one of them cannot be ignored. Numerical method was adopted and parameters of the hydrogen diffusion of the specimen had been compared. It can be observed that different stress type have different impact on the hydrogen diffusion. The tensile stress will promote the hydrogen diffusion but the pressure stress will hinder the hydrogen diffusion. According to Sievert's law, with hydrogen concentration increase or decrease, the internal pressure of hydrogen induced crack changes accordingly.

\section{Acknowledgments}

The authors wish to thank the Administration of Quality Supervision, Inspection and Quarantine science and technology plan projects(2014QK172) and Sinopec Zhenhai Refining \& Chemical Company (2014KF002) scientific research project for financial support of this work.

\section{References}

[1] Bosch C, Delafosse D, Longaygue X. Effects of strain and trapping on hydrogen-induced cracking in high strength low alloy steels. In: European corrosion congress, vol. 3; 2010. p. 2259-2269.

[2] Jin TY, Liu ZY, Cheng YF. Effect of non-metallic inclusions on hydrogen-induced cracking of api5l x100 steel. Int J Hydrogen Energy 2010;35(15):8014-8021.

[3] Michler T, Lee Y, Gangloff RP, Naumann J. Influence of macro segregation on hydrogen environment embrittlement of sus $316 \mathrm{l}$ stainless steel. Int J Hydrogen Energy 2009;34(7): 3201-3209.

[4] Brouwer RC. Modelling hydrogen induced crack growth in the wall of pipelines and pressure vessels. In: Proceedings of ECF, vol. 10; 1994.

[5] Anderson TL. Fracture mechanics fundamentals and applications. CRC Press; 2005.

[6] Api standard 579-1/asme ffs-1. Fitness-for-service standard; 2007.

[7] Jiang WC, Gong JM. Finite element simulation of hydrogen blistering of steel 16MnR serving in wet H2S environment. Journal of Jilin University (Engineering and Technology Edition) 2008;38(1):61-65. 